\title{
Sensitization of an Endogenous Photosensitizer: Electronic Spectroscopy of Riboflavin in the Proximity of Semiconductor, Insulator, and Metal Nanoparticles
}

\author{
Siddhi Chaudhuri, ${ }^{\dagger}$ Samim Sardar, ${ }^{\dagger}$ Damayanti Bagchi, ${ }^{\dagger}$ Shib Shankar Singha, ${ }^{\ddagger}$ Peter Lemmens, ${ }^{\S, \perp}$ \\ and Samir Kumar Pal* ${ }^{\dagger}$
}

\begin{abstract}
${ }^{\dagger}$ Department of Chemical, Biological and Macromolecular Sciences, S. N. Bose National Centre for Basic Sciences, Block JD, Sector III, Salt Lake, Kolkata 700 098, India

${ }^{\ddagger}$ Department of Physics, Bose Institute, 93/1, Acharya Prafulla Chandra Road, Kolkata 700 009, India

${ }^{\S}$ Institute for Condensed Matter Physics and ${ }^{\perp}$ Laboratory for Emerging Nanometrology, TU Braunschweig, Mendelssohnstraße 3, 38106 Braunschweig, Germany
\end{abstract}

\begin{abstract}
Riboflavin (Rf) is a class of important vitamins (Vitamin $\mathrm{B}_{2}$ ) and a well-known antioxidant. Here we have synthesized nanohybrids of $\mathrm{Rf}$ with a number of inorganic nanoparticles (NPs); namely zinc oxide $(\mathrm{ZnO})$, titanium oxide $\left(\mathrm{TiO}_{2}\right)$, aluminum oxide $\left(\mathrm{Al}_{2} \mathrm{O}_{3}\right)$ and gold NPs of similar sizes. While high resolution transmission electron microscopy (HRTEM) confirms integrity and sizes of the NPs, intactness of the molecular structure of the drug $\mathrm{Rf}$ is revealed from absorption and steady-state emission spectra of the drug in the nanohybrid. Raman spectroscopy on the nanohybrids shows the nature of molecular complexation of the drug with the inorganic NPs. For the semiconductor and insulator NPs, the complexation is found to be noncovalent, however, a covalent
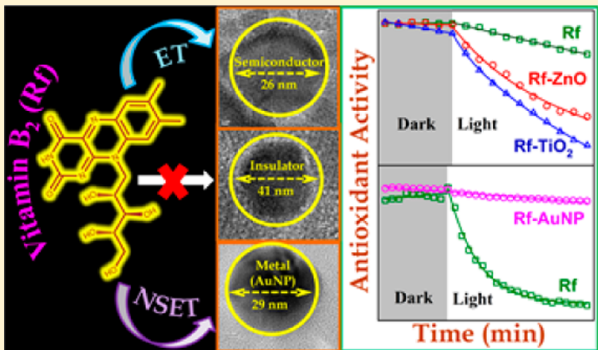
attachment of the drug with the dangling bonds of metal atoms at the surface is observed. In order to investigate antioxidant activity of the nanohybrids, we have performed 2, 2-diphenyl-1-picrylhydrazyl (DPPH) assay of the nanohybrids in dark as well as under blue light irradiation. Whereas change of the antioxidant activity of the nanohybrids with respect to free riboflavin in the absence of light is observed to be insignificant, a drastic change in the activity in the case of $\mathrm{TiO}_{2}$ and $\mathrm{ZnO}$ in the presence of light is evident. No change in the case of $\mathrm{Al}_{2} \mathrm{O}_{3}$ and a significant decrease in the antioxidant activity for gold nanohybrids are also remarkable. Picosecond-resolved fluorescence studies on the nanohybrids reveal a molecular picture of the differential antioxidant activities. An ultrafast photoinduced electron transfer from $\mathrm{Rf}$ to $\mathrm{ZnO}$ and $\mathrm{TiO}_{2}$ are clearly evident from the corresponding fluorescence transients. We have compared the picosecond-resolved transients with that of $\mathrm{Rf}$ in the presence of a well-known electron acceptor benzoquinone (BQ) and found similar time scales. No temporal change in the fluorescence transient of riboflavin in $\mathrm{Al}_{2} \mathrm{O}_{3}$ nanohybrids compared to that of free $\mathrm{Rf}$ is observed indicating uneventful excited state relaxation of the nanohybrids. Nanosurface energy transfer (NSET) over Förster resonance energy transfer (FRET) is found to be the prevailing de-excitation mechanism in the case of gold nanohybrids, because of the strong spectral overlap between Rf emission and surface plasmon absorption of the gold NPs. Different excited state mechanisms as revealed from our studies are expected to be useful for the design of NP-sensitized drugs, which are reported sparsely in the literature.
\end{abstract}

\section{INTRODUCTION}

Riboflavin (Rf) or vitamin $B_{2}$ is an essential micronutrient and substantially present in dietary products such as vegetables, mushrooms, yeast, milk, cheese, eggs, and meat. ${ }^{1-3}$ Although remarkable medicinal use of the vitamin as a food supplement followed immediately after the Nobel-winning (in chemistry, 1938) structural determination of the micronutrient, alternative use of the vitamin as a potential photodynamic therapy (PDT) agent is also evident in early ${ }^{4}$ as well as in contemporary ${ }^{5}$ literature. Upon light irradiation of a specific wavelength, a photosensitizer absorbs photon and elevated from ground state to an excited singlet state which in due course decays to the triplet excited state via intersystem crossing (ISC). Then the triplet excited state energy is transferred to molecular oxygen at ground state to produce singlet oxygen, which is a central intermediate for cytotoxic action in PDT. It is well established that $\mathrm{Rf}$ upon visible light irradiation generates reactive oxygen species (ROS) such as singlet oxygen and superoxide anion. ${ }^{6,7}$ Riboflavin photosensitization has been used as a therapy for the treatment of keratosis, where the vitamin is an endogenous photosensitizer. $^{8,9}$

In the past two decades, nanoparticle- (NP-) based therapeutic products have flourished for the enhancement of drug action. ${ }^{10-13}$ Nanomaterials are attributed with distinguished physicochemical properties, such as very small size, greater surface area to mass ratio, and high reactivity, which is

Received: March 30, 2015

Revised: April 13, 2015

Published: April 14, 2015 
different from that of bulk materials of similar composition. ${ }^{11}$ Nanomaterials as drug carriers can overcome some of the constraints such as low solubility and diffusivity, shortened blood circulation half-life, high immunogenicity and low bioavailability found in conventional therapeutic and diagnostic agents. ${ }^{11,14}$ Because of a higher surface area to volume ratio, nanocarriers show upgraded pharmacokinetics and bio distribution of therapeutic agents and reduce toxicity by their accumulation at the target site. ${ }^{15}$ The solubility of hydrophobic drugs increases by the formation of nanoconjugates and render them convenient for parenteral administration. Moreover, the stability of various therapeutic agents such as peptides and oligonucleotides increases which can be utilized to conduct the drug to central nervous system due to their limited size and greater barrier permeability. ${ }^{15,16}$ However, reports on sensitization of drugs by NPs through their electronic coupling are sparse in the literature. On the other hand, formation of nanohybrid of a drug with an inorganic nanoparticle is the key for the modulation of drug activity in the nanohybrid. In one of our earlier reports, we have explored the photoinduced ultrafast dynamics in a well-known cancer drug, protoporphyrin IX (PP) sensitized with $\mathrm{ZnO}$ NPs ( $\mathrm{PP}-\mathrm{ZnO}$ ). We successfully showed that the $\mathrm{PP}-\mathrm{ZnO}$ nanohybrid exhibits enhanced activity in PDT compared to that of only PP. Zinc oxide NPs act as drug delivery vehicle and also facilitate the charge separation which eventually enhances the drug activity. ${ }^{17}$

In the present study, we have synthesized nanohybrids of vitamin $\mathrm{B}_{2}$ with various inorganic NPs such as zinc oxide $(\mathrm{ZnO})$, titanium oxide $\left(\mathrm{TiO}_{2}\right)$, aluminum oxide $\left(\mathrm{Al}_{2} \mathrm{O}_{3}\right)$, and gold NPs of approximately $30 \mathrm{~nm}$ sizes. We confirmed the sizes of the NPs using high resolution transmission electron microscopy (HRTEM). Raman spectroscopic studies provide an insight on the nature of the attachment of riboflavin to the surfaces of the semiconductor $\left(\mathrm{TiO}_{2}\right.$ and $\left.\mathrm{ZnO}\right)$, the insulator $\left(\mathrm{Al}_{2} \mathrm{O}_{3}\right)$ NPs and metal (gold NPs). In order to measure the photoantioxidant activity of the nanohybrids, we have performed the well-known 2,2-diphenyl-1-picrylhydrazyl $(\mathrm{DPPH})$ assay under blue light irradiation $\left(\lambda_{\max }=450 \mathrm{~nm}\right)$. Time-resolved fluorescence studies on the nanohybrids were carried out to understand the electron transfer from photoexcited $\mathrm{Rf}$ to $\mathrm{ZnO}$ or $\mathrm{TiO}_{2}$, which eventually intensifies the ROS activity in the $\mathrm{Rf}-$ semiconductor nanostructures. The phenomenon of nano surface energy transfer (NSET) is predominant in the nanohybrids of riboflavin with gold. Our studies unravel a mechanistic pathway of drug sensitization with various inorganic NPs to determine the photoinduced antioxidant property of this important vitamin.

\section{MATERIAL AND METHODS}

Chemicals used for synthesis in this study are of analytical grades and without further purification. Approximately $30 \mathrm{~nm}$ $\mathrm{ZnO} \mathrm{NPs}, \mathrm{TiO}_{2} \mathrm{NPs}, \mathrm{Al}_{2} \mathrm{O}_{3} \mathrm{NPs}$, riboflavin, gold(III) chloride, and sodium citrate were purchased from Sigma-Aldrich. The suitable solvent for $p$-benzoquinone (BQ Fluka) was ethanol (Merck). Millipore water was used as aqueous solution.

2.1. Synthesis of Riboflavin Nanohybrids with $\mathrm{ZnO}$ NPs, $\mathrm{TiO}_{2} \mathrm{NPs}$, and $\mathrm{Al}_{2} \mathrm{O}_{3} \mathrm{NPs}$. A $20 \mu \mathrm{M}$ Rf solution was prepared in alcohol. Then $0.5 \mathrm{mg} / \mathrm{mL}$ of solid $30 \mathrm{~nm} \mathrm{ZnO}$, $\mathrm{TiO}_{2}$, and $\mathrm{Al}_{2} \mathrm{O}_{3}$ were added to prepared $\mathrm{Rf}$ solution and were stirred constantly for $12 \mathrm{~h}$. The sensitization of $\mathrm{ZnO} \mathrm{NPs}, \mathrm{TiO}_{2}$ $\mathrm{NPs}$, and $\mathrm{Al}_{2} \mathrm{O}_{3} \mathrm{NPs}$ with $\mathrm{Rf}$ dye was performed at room temperature in the dark. After the sensitization process, the solution was centrifuged for a while and the clear supernatant solution of unattached dyes was decanted. Then the nanohybrids were washed with alcohol 3-4 times. Then the samples were dried in a water bath. The $\mathrm{Rf}$ concentration in the nanohybrids was $3 \mu \mathrm{M}$.

2.2. Synthesis of Riboflavin-Gold Nanohybrids. A 10 $\mathrm{mL} 80 \mu \mathrm{M}$ Rf solution was prepared in water. Then $100 \mathrm{mM}$ stock solution of $\mathrm{AuCl}_{3}$ was prepared, and $50 \mu \mathrm{L}$ of $\mathrm{Au}$ (III) chloride solution was mixed with $10 \mathrm{~mL}$ of the stock riboflavin solution. The mixture was kept under UV-light $\left(\lambda_{\max }=365 \mathrm{~nm}\right)$ for $15 \mathrm{~min}$ until the characteristic surface plasmon resonance band was observed at $540 \mathrm{~nm} .{ }^{18}$ Here, a different technique is used to remove free riboflavin from Rf-AuNPs. Since it is difficult to retrieve Rf-AuNPs after centrifugation, the excess dye was driven out by dialysis prior to the experiments. The $\mathrm{Rf}$ and AuNPs concentrations in the nanohybrids were $40 \mu \mathrm{M}$ and $0.1 \mathrm{nM}$, respectively.

2.2. Synthesis of Citrate-Capped Gold Nanoparticles. First a $0.5 \mathrm{M}$ stock solution of sodium citrate and $100 \mathrm{mM}$ stock solution of $\mathrm{AuCl}_{3}$ were prepared in water. A $20 \mu \mathrm{L}$ aliquot of stock sodium citrate was added to $4 \mathrm{~mL}$ of $0.5 \mathrm{mM}$ of $\mathrm{AuCl}_{3}$ solution while boiling it. The solution turned reddish in color. The formation of gold NPs was confirmed by the surface plasmon resonance band at $540 \mathrm{~nm} .{ }^{19}$

2.3. Optical Studies. Shimadzu UV-2600 spectrophotometer was used to measure absorption spectra using a quartz cell of $1 \mathrm{~cm}$ path length. The extinction coefficient values of 12200 $\mathrm{M}^{-1} \mathrm{~cm}^{-1}$ at $450 \mathrm{~nm}$ for riboflavin (Rf) ${ }^{20}$ and $2.93 \times 10^{9}$ for citrate-capped AuNPs ${ }^{21}$ were used to calculate the concentration of the samples. The extinction coefficient of riboflavin and its nanoconjugates were measured to be same and thus the similar value was used for calculation purpose. The degradation kinetics under blue light was measured with spectroscopic precision using an indigenously developed fiber-optic based system which is generally used for measuring sensitive optical measurements. ${ }^{22} \mathrm{DPPH}$ has a characteristic absorbance maximum at $520 \mathrm{~nm}$. This characteristic absorption peak was chosen to monitor the DPPH degradation under blue light irradiation at room temperature. Spectra suite software from Ocean Optics detected the absorbance of DPPH at $30 \mathrm{~s}$ intervals and recorded them. Finally, the DPPH absorbance at $520 \mathrm{~nm}$ was plotted as a function of time. The experiments on steady-state fluorescence measurements were performed using JobinYvon Fluorolog fluorometer. The emission of $\mathrm{Rf}$ along with its conjugates were taken upon excitation at the wavelength of $409 \mathrm{~nm}$. Rf and DPPH concentrations were $0.1 \mu \mathrm{M}$ and $10 \mu \mathrm{M}$ respectively. The fluorescence measurements were performed with the excitation and emission bandwidth slits of $2 \mathrm{~nm}$. The equation used to calculate the quantum yield is $Q=\left(Q_{R}\left(I / I_{R}\right)\left(O D_{R} / O D\right)\left(n^{2} / n_{R}^{2}\right)\right)$, where the quantum yields of the reference and the $R f$ are $Q_{R}$ and $Q$ the integrated fluorescence intensities of $\mathrm{Rf}$ and the reference are $I$ and $I_{R}$ at the excitation wavelength, the absorbance of Rf and the reference are $\mathrm{OD}$ and $\mathrm{OD}_{\mathrm{R}}$; and the refractive indices of $\mathrm{Rf}$ and the reference solutions are $n$ and $n_{\mathrm{R}}{ }^{23}$ The reported absolute quantum yield of $\mathrm{Rf}$ in ethanol is $0.3{ }^{24}$ The Raman measurements were performed in a micro Raman set up which consists of spectrometer (Lab RAM HR, Jovin Yvon) with a Peltier-cooled CCD detector. A He-Ne laser with wavelength $633 \mathrm{~nm}$, objective 10X, integration time $20 \mathrm{~s}$ and a power of 6 $\mathrm{mW}$ was used as excitation. A time correlated single photon counting (TCSPC) from Edinburgh Instruments was used in performing the time-resolved fluorescence spectroscopic experiments. A picosecond pulsed laser diode having instrument 
response function (IRF) of 80 ps was used for excitation of the sample at $409 \mathrm{~nm}$. The emission was monitored through a polarizer oriented at $55^{\circ}$ from the vertical position and the excitation was vertically polarized. The multiexponential (n) function, $\sum_{i=1}^{n} A_{i} \exp \left(-t / \tau_{i}\right)$ is used to fit the fluorescence transients, where, $A_{i}$ 's are weighted percentages with time constants of $\tau_{i}$. The equation $\tau=\sum_{i=1}^{n} A_{i} \tau_{i}$, when $\sum_{i=1}^{n} A_{i}=1$ expresses the average lifetime at excited state.

2.4. Förster's Resonance Energy Transfer (FRET) Technique. The technique is discussed in our previous studies. ${ }^{3,17}$ In brief, the donor-acceptor distances were derived using the equation, $R_{0}=0.211 \times\left[\kappa^{2} n^{-4} \Phi_{D} J(\lambda)\right]^{(1 / 6)}$ in $\AA$. $R_{0}$ is assigned as the donor-acceptor distance at $50 \%$ energy transfer efficiency, $\kappa^{2}$ is the relative orientation of the donor-acceptor transition dipoles in space. The magnitude of $\kappa^{2}$ is 0.66 for random orientation of donor-acceptor. The refractive index $(n)$ of the medium is $1.33 . J(\lambda)$ is the spectral overlap between the donor emission and the acceptor absorption. It is denoted by,

$$
J(\lambda)=\frac{\int_{0}^{\infty} F_{D}(\lambda) \varepsilon_{A}(\lambda) \lambda^{4} \mathrm{~d} \lambda}{\int_{0}^{\infty} F_{D}(\lambda) \mathrm{d} \lambda}
$$

where, $F_{\mathrm{D}}(\lambda)$ is the donor fluorescence intensity in the wavelength range of $\lambda$ to $\lambda+\mathrm{d} \lambda$ and is dimensionless. $\varepsilon_{\mathrm{A}}(\lambda)$ is the acceptor extinction coefficient (in $\mathrm{M}^{-1} \mathrm{~cm}^{-1}$ ) at $\lambda$. After knowing the $R_{0}$ value, the $r$ is determined from the equation

$$
r^{6}=\left[R_{0}{ }^{6}(1-E)\right] / E
$$

where, $E$ is the efficiency of energy transfer, which can be deduced as

$$
E=1-\frac{\tau_{D A}}{\tau_{D}}
$$

where, $\tau_{D}$ and $\tau_{D A}$ are donor lifetimes with and without acceptor. ${ }^{23}$

2.5. Nanosurface Energy Transfer (NSET) Technique. The technique is explained in our previous studies. ${ }^{25}$ This technique is based on the model of Persson and Lang. ${ }^{26}$ The distances of donor-acceptor pair were calculated using the following equations.

$$
k_{e t}=0.3\left(\frac{\mu^{2} \omega_{d y e}}{\eta \omega_{F} k_{F} d^{4}}\right)
$$

which can be expressed in more measurable parameters through the use of the Einstein $A_{21}$ coefficient. $^{27}$

$$
A_{21}=\frac{\omega_{d y e}{ }^{3}}{3 \varepsilon_{0} \eta \pi c^{3}}\left|\mu^{2}\right|
$$

The rate of energy transfer in accordance with Coulomb's law $\left((1 / 4) \pi \varepsilon_{0}\right)$ is expressed as

$$
k_{e t}=0.225 \frac{c^{3} \phi_{d y e}}{\omega_{d y e}{ }^{2} \omega_{F} k_{F} d^{4} \tau_{d y e}}
$$

where $c$ denotes the speed of light and the quantum yield $\left(\varphi_{\text {dye }}\right)$ of the donor is 0.26 . The angular frequency $\left(\omega_{\text {dye }}\right)$ for the donor is $4.1 \times 10^{15} \mathrm{~s}^{-1}$ and the angular frequency $\left(\omega_{\mathrm{F}}\right)$ for bulk gold is $8.4 \times 10^{15} \mathrm{~s}^{-1}$. $\mathrm{d}$ is the donor-acceptor separation. The average lifetime of the dye $\left(\tau_{\text {dye }}\right)$ is $4760(\mathrm{ps})$, and the Fermi wavevector $\left(\mathrm{k}_{\mathrm{F}}\right)$ for bulk gold is $1.2 \times 10^{8} \mathrm{~cm}^{-1}$. The $\mathrm{d}_{0}$ value is the distance at which a dye exhibits equal probabilities for energy transfer and spontaneous emission. The $d_{0}$ value is given by,

$$
d_{0}=\left(0.225 \frac{c^{3} \phi_{d y e}}{\omega_{d y e}^{2} \omega_{F} k_{F}}\right)^{1 / 4}
$$

Here, we have used $k_{\text {et }}$ as $k_{\text {time-resolved }}$

$$
k_{\text {time-resolved }}=\frac{1}{\tau_{\text {donor-acceptor }}}-\frac{1}{\tau_{\text {dye }}}
$$

where $\tau_{\text {donor-acceptor }}$ depicts the average donor-acceptor lifetime.

\section{RESULTS AND DISCUSSION}

Figure 1a shows a high-resolution transmission electron microscopic (HR-TEM) image of $\mathrm{ZnO}$ NP. Lattice fringes of $\mathrm{ZnO} \mathrm{NP}$ is found to be $\sim 0.26 \mathrm{~nm}$ which corresponds to the distance between two (002) planes. ${ }^{17}$ The average particle size is found to be $\sim 28.5 \pm 0.4 \mathrm{~nm}$ from experimental TEM data. Figure $1 \mathrm{~b}$ and $1 \mathrm{c}$ show lattice fringes of $\mathrm{TiO}_{2}$ and $\mathrm{Al}_{2} \mathrm{O}_{3}$ with
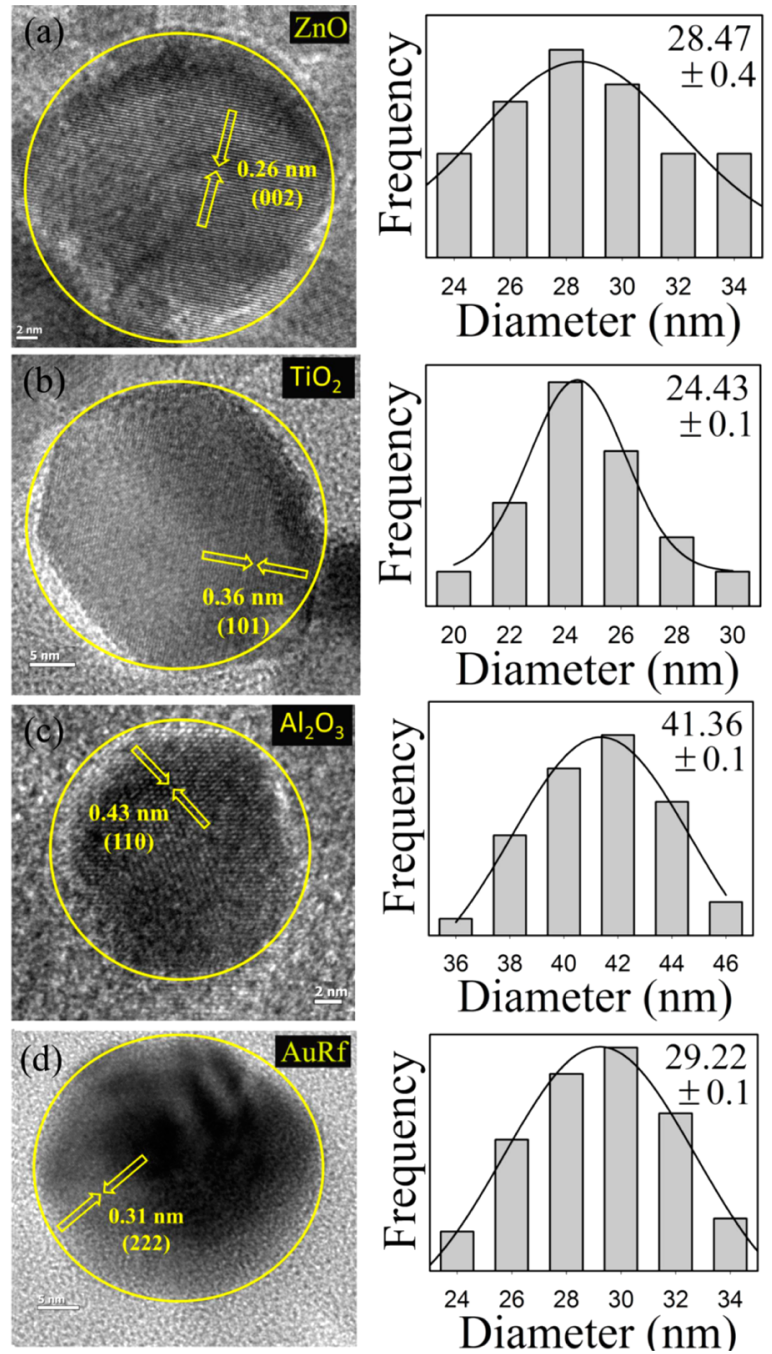

Figure 1. (a-d) HRTEM images of various nanoparticles. The right panel shows the corresponding particle size distribution of the NPs. 
interplanar distances $0.36 \mathrm{~nm}$ between two (101) planes and $0.42 \mathrm{~nm}$ between two (110) planes, ${ }^{28,29}$ respectively. The average particle size is estimated to be $\sim 24.4 \pm 0.1 \mathrm{~nm}$ for $\mathrm{TiO}_{2}$ and $\sim 41.4 \pm 0.1 \mathrm{~nm}$ for $\mathrm{Al}_{2} \mathrm{O}_{3}$. From the HRTEM images, $\mathrm{TiO}_{2}$ NPs seem to be faceted with different crystal planes. However, careful investigation on the interfringe distances, for a number of NPs reveals a distance of $0.36 \mathrm{~nm}$ which is consistent with (101) plane of $\mathrm{TiO}_{2}$ crystal. Thus, surface roughness of the $\mathrm{TiO}_{2} \mathrm{NPs}$ is concluded to be responsible for the faceted appearance of the NPs. The lattice fringes of $\mathrm{Rf}-\mathrm{AuNP}$ (Figure 1d) distinctly show an interplanar distance of $\sim 0.31 \mathrm{~nm},{ }^{21}$ corresponding to the interspace between two (222) planes. The average particle size for RfAuNP is found to be $\sim 29.2 \pm 0.1 \mathrm{~nm}$.

$\mathrm{UV}$-visible spectroscopy is a useful technique to understand the complexation between Rf and the NPs. ${ }^{30}$ The absorption maxima of $\mathrm{Rf}$ is at 360 and $445 \mathrm{~nm}$ in water and ethanol, respectively. The absorption, emission and excitation spectra of $\mathrm{Rf}$ with its nanohybrids are shown in Figure $2 \mathrm{a}-\mathrm{c}$, respectively, in water for gold nanohybrids and in ethanol for the rest. The $\mathrm{Rf}$ absorption in the presence of different NPs are shown in Figure 2a. The change in absorbance peak at $445 \mathrm{~nm}$ is due to
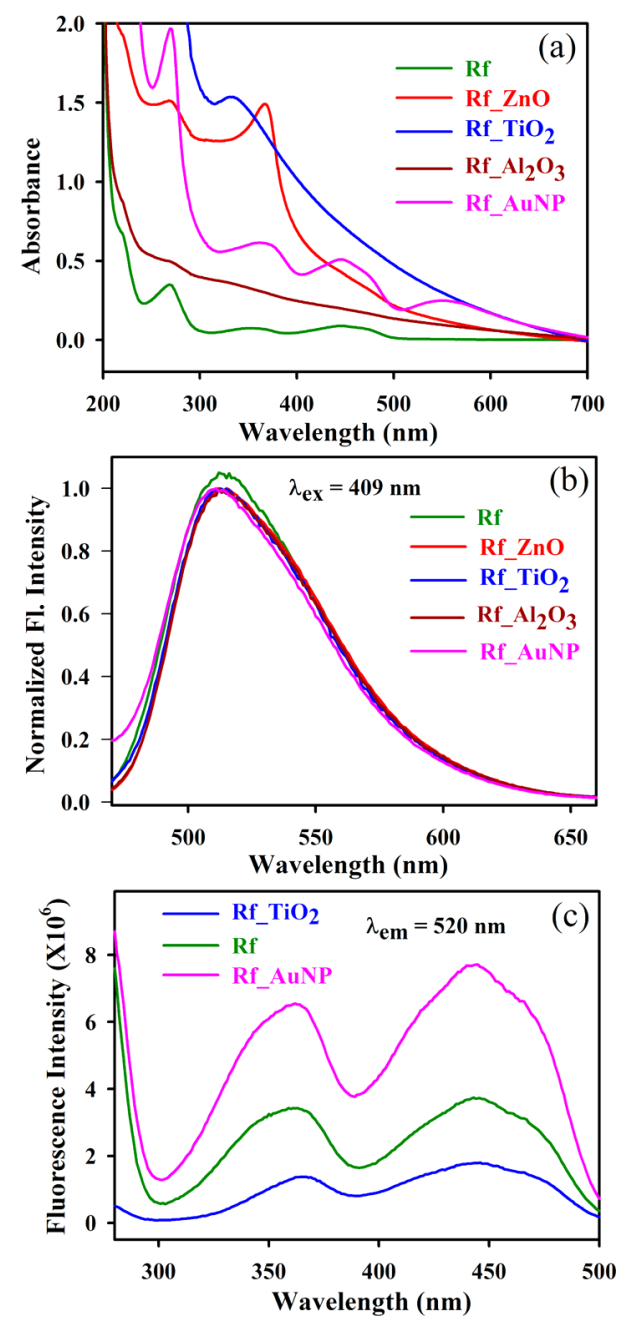

Figure 2. (a) Absorption, (b) emisssion and (c) excitation spectra of $\mathrm{Rf}$ in ethanol and $\mathrm{Rf}-$ nanocomposites $\left(\mathrm{Rf}-\mathrm{ZnO}, \mathrm{Rf}-\mathrm{TiO}_{2}\right.$, and $\mathrm{Rf}-$ $\mathrm{Al}_{2} \mathrm{O}_{3}$ in ethanol and $\mathrm{Rf}-\mathrm{AuNP}$ in water). The excitation spectra of $\mathrm{Rf}$ along with its various nanohybrids show no relative change in the peak position. For the clarity three representative spectra were shown. complex formation when $\mathrm{Rf}$ attaches to surfaces of $\mathrm{ZnO}, \mathrm{TiO}_{2}$ and $\mathrm{Al}_{2} \mathrm{O}_{3}$ NPs. The observation is well documented in the literature. ${ }^{17,28}$ No distinct peak of $\mathrm{Rf}$ is observed in these nanohybrids due to the very low riboflavin concentration and large scattering of the nanoparticles. However, the presence of riboflavin is evident in emission and excitation spectrum of Figure 2, parts $b$ and $c$, respectively. The concentration of riboflavin is estimated by differential spectra method (data not shown). However, a surface plasmon band (SPB) exists in RfAuNP due to the contribution of AuNPs. The SPB exists as a result of collective oscillations of the electron at the NP surface (6s electrons for AuNPs) which is correlated with the electromagnetic field of the incoming light. ${ }^{31}$ From the normalized steady-state emission data of $\mathrm{Rf}$ with its various nanohybrids ( $\mathrm{Rf}-\mathrm{ZnO}, \mathrm{Rf}-\mathrm{TiO}_{2}$, and $\mathrm{Rf}-\mathrm{Al}_{2} \mathrm{O}_{3}$ in ethanol and $\mathrm{Rf}$-AuNPs in water), no change in peak position is observed. Figure $2 \mathrm{c}$ shows the corresponding excitation spectra.

The Raman spectra of $\mathrm{Rf}$-nanohybrids with $\mathrm{ZnO}, \mathrm{TiO}_{2}$, and $\mathrm{Al}_{2} \mathrm{O}_{3}$ NPs show similar vibrational modes as that of the corresponding NPs (as shown in Figure $3 b$ ) which proves the good retention of crystal structure of the NPs after the complex formation. These studies reveal no change in the peak positions of the NPs. Additionally, the characteristic Raman peaks for Rf in the nanohybrids show insignificant perturbation which indicates the absence of strong chemical interaction of $\mathrm{Rf}$ with
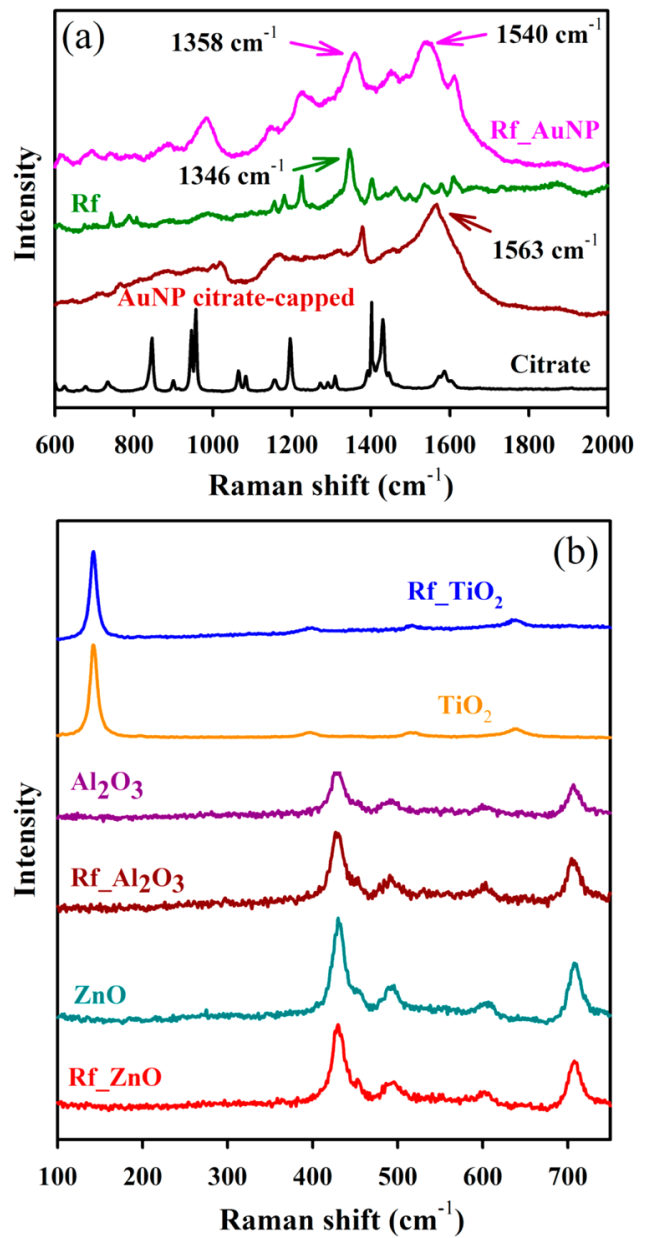

Figure 3. Raman spectra of (a) Rf and Rf-AuNPs in solid phase and (b) different nanoparticles and their nanohybrids with $\mathrm{Rf}$ in solid phase. 
the NPs. The observation is consistent with the reported literature. The invariance of the peak positions of $\mathrm{Rf}-$ nanohybrids suggests that the complexation is mainly due to physisorption. ${ }^{30}$ However, when Rf is attached to gold NP the characteristic Rf peak is shifted from 1346 to $1358 \mathrm{~cm}^{-1}$ and also broadened (Figure 3a). Moreover, the peak at $1563 \mathrm{~cm}^{-1}$ for AuNPs is moved to $1540 \mathrm{~cm}^{-1}$ in the presence of $\mathrm{Rf}$ molecules. This suggests a covalent bond formation between $\mathrm{Rf}$ and $\mathrm{Au} \mathrm{NP} .^{32}$

Figure 4a demonstrates antioxidant activity of the $\mathrm{Rf}$ nanohybrids with colloidal $\mathrm{ZnO}$ and $\mathrm{TiO}_{2} \mathrm{NPs}$ in alcohol
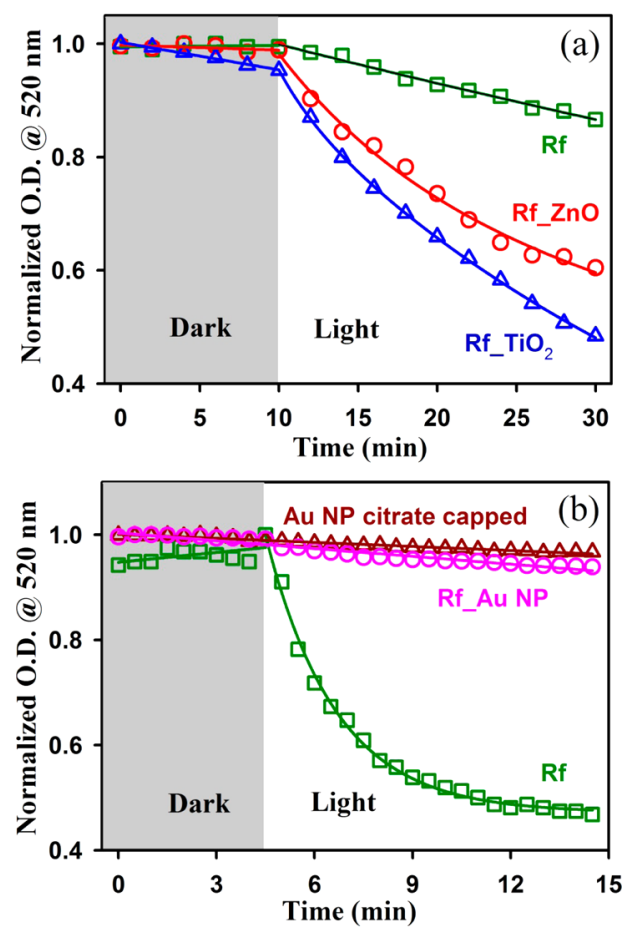

Figure 4. Absorption kinetics (at $520 \mathrm{~nm}$ ) of DPPH in the presence of (a) $\mathrm{Rf}-\mathrm{ZnO}, \mathrm{Rf}-\mathrm{TiO}_{2}$ in ethanol and (b) $\mathrm{Rf}-\mathrm{AuNPs}$ in ethanol:water $(1: 1)$ mixture under blue light irradiation. Note that $\mathrm{Rf}$ concentration is ten times more in $\mathrm{Rf}-\mathrm{AuNP}$ than that in $\mathrm{Rf}-\mathrm{ZnO}$ and $\mathrm{Rf}-\mathrm{TiO}_{2}$. Thus, the control $\mathrm{Rf}$ in the lower panel shows enhanced activity than the control Rf (upper panel). Part b shows the degradation kinetics under dark only up to $5 \mathrm{~min}$ for the representation as antioxidant activity under dark for longer time window of $10 \mathrm{~min}$ shows no significant activity.

without and with light exposure, respectively. Antioxidant activities of the nanohybrids as well as that of the vitamin are usually monitored by the decolourization kinetics of a stable free radical 2, 2-diphenyl-1-picrylhydrazyl (DPPH). The vitamin or nanohybrids donate a proton/electron to the violet-colored radical DPPH which reduces the radical to $\mathrm{DPPH}_{2}$ form which is yellow in color. ${ }^{33}$ The free radical quenching kinetics of $\mathrm{Rf}, \mathrm{Rf}-\mathrm{ZnO}$, and $\mathrm{Rf}-\mathrm{TiO}_{2}$ under blue light irradiation have been fitted with biexponential decay functions. The time constants are calculated to be 140,50 , and $26 \mathrm{~min}$, respectively. The increase in the rate of radical scavenging activity of the vitamin attached to semiconductor NPs under blue light is clearly evident from Figure 4a. Moreover, there is no change in the activity of $\mathrm{Rf}-\mathrm{Al}_{2} \mathrm{O}_{3}$ nanohybrids (data not shown here) compared to $\mathrm{Rf}$ in ethanol solution. Hence, the rate of photoinduced antioxidant activity in the presence of light is highly dependent on the nature of the nanoparticles. Figure $4 \mathrm{~b}$ illustrates that the antioxidant activity of the Rf-AuNP nanohybrids is mostly suppressed compared to that of the solution of $\mathrm{Rf}$ only. The antioxidant activities of $\mathrm{Rf}, \mathrm{Rf}-\mathrm{ZnO}$ and $\mathrm{Rf}-\mathrm{TiO}_{2}$ as shown in Figure $4 \mathrm{a}$ were performed in ethanol. The antioxidant activities in Figure $4 \mathrm{~b}$ were carried out in ethanol-water (1:1) mixture. As mentioned earlier Rf-AuNPs are synthesized in aqueous phase and the free radical DPPH is soluble in ethanol. It has to be noted that $\mathrm{Rf}$ concentration is ten times more in Rf-AuNP than that in $\mathrm{Rf}-\mathrm{ZnO}$ and $\mathrm{Rf}-\mathrm{TiO}_{2}$ Thus, the control $\mathrm{Rf}$ in Figure $4 \mathrm{~b}$ shows enhanced activity than the control $R f$ in Figure 4a.

In order to explain the above observations based on the $\mathrm{DPPH}$ assay of the $\mathrm{Rf}-$ nanohybrids, picosecond resolved fluorescence spectroscopy measurments were performed. The fluorescence decay profiles for free $\mathrm{Rf}, \mathrm{Rf}-\mathrm{ZnO}$, and $\mathrm{Rf}-\mathrm{TiO}_{2}$ were obtained upon excitation at $409 \mathrm{~nm}$ in ethanol and monitored at $520 \mathrm{~nm}$ as shown in Figure 5a. The time
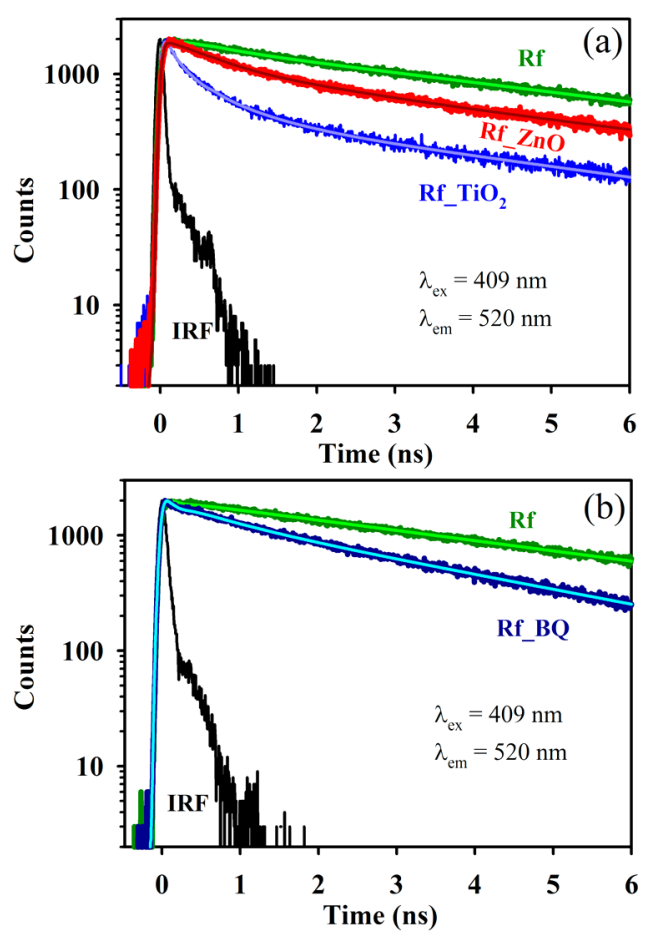

Figure 5. Picosecond-resolved fluorescence transients of $\mathrm{Rf}$ (excitation at $409 \mathrm{~nm}$ ) in absence and presence of $(\mathrm{a}) \mathrm{ZnO}, \mathrm{TiO}_{2}$ and (b) $\mathrm{BQ}$ in ethanol.

constants of the fluorescence transients at $520 \mathrm{~nm}$ for the singlet excited-state of $\mathrm{Rf}$ in ethanol solution showed a lifetime of $3.4 \mathrm{~ns}$ as shown in Table 1 . The quenching of the lifetime transients is observed in $\mathrm{Rf}-\mathrm{ZnO}$ and $\mathrm{Rf}-\mathrm{TiO}_{2}$ and no change in lifetime is observed in $\mathrm{Rf}-\mathrm{Al}_{2} \mathrm{O}_{3}$. The observation is consistent with the fact that the excited vitamin transfers electrons to the semiconductor NPs. ${ }^{34-36}$ The lifetime components of the transients are tabulated in Table 1. In order to confirm the electron migration from Rf, the wellknown electron acceptor benzoquinone (BQ) is bound to $\mathrm{Rf}$ and the possible excited-state interactions in Rf-BQ composite is investigated with maximum $\mathrm{BQ}$ concentration of $10 \mu \mathrm{M}$ (Figure $5 \mathrm{~b})$. The electron transfer time constant $(\sim 100 \mathrm{ps})$ from $\mathrm{Rf}$ to $\mathrm{BQ}$ are found to be similar to that of $\mathrm{Rf}-\mathrm{ZnO}$ and $\mathrm{Rf}-\mathrm{TiO}_{2}$ (Table 1). This corroborates with the photoinduced antioxidant activity data (Figure 4a). The enhancement in the 
Table 1. Dynamics of Picosecond-Resolved Fluoresence Transients of Rf and Rf-Nanohybrids ${ }^{a}$

\begin{tabular}{|c|c|c|c|c|c|c|}
\hline sample & excitation wavelength $(\mathrm{nm})$ & detection wavelength $(\mathrm{nm})$ & $\tau_{1}(\mathrm{~ns})$ & $\tau_{2}(\mathrm{~ns})$ & $\tau_{3}(\mathrm{~ns})$ & $\tau_{\text {avg }}(\mathrm{ns})$ \\
\hline $\mathrm{Rf}$ in ethanol & 409 & 520 & $0.11(32 \%)$ & $4.89(68 \%)$ & - & 3.40 \\
\hline $\mathrm{Rf}-\mathrm{ZnO}$ nanohybrid & 409 & 520 & $0.44(47 \%)$ & $2.19(23 \%)$ & $6.41(7.2 \%)$ & 2.63 \\
\hline $\mathrm{Rf}-\mathrm{TiO}_{2}$ nanohybrid & 409 & 520 & $0.15(65 \%)$ & $0.65(21 \%)$ & $4.72(14 \%)$ & 0.89 \\
\hline $\mathrm{Rf}-\mathrm{BQ}$ & 409 & 520 & $0.05(3 \%)$ & $0.72(27 \%)$ & $3.34(70 \%)$ & 2.56 \\
\hline $\mathrm{Rf}$ in water & 409 & 520 & $4.76(100 \%)$ & & & 4.75 \\
\hline $\mathrm{Rf}-\mathrm{AuNP}$ in water & 409 & 520 & $0.40(48 \%)$ & $4.65(52 \%)$ & & 2.49 \\
\hline
\end{tabular}

${ }^{a}$ Numbers in the parentheses indicate relative weightages.

photoinduced antioxidant activity in $\mathrm{Rf}-\mathrm{ZnO}$ and $\mathrm{Rf}-\mathrm{TiO}_{2}$ nanohybrids is due to efficient charge separation created by illumination with light in the nanohybrids with wide-band gap $(\sim 3.0-3.2 \mathrm{eV})$. On the other hand, the photoinduced antioxidant activity of Rf-AuNPs is diminished (Figure $4 \mathrm{~b}$ ). This suggests that the vitamin is de-excited by transferring its nonradiative energy to the gold nanoparticles. Figure $6 a$
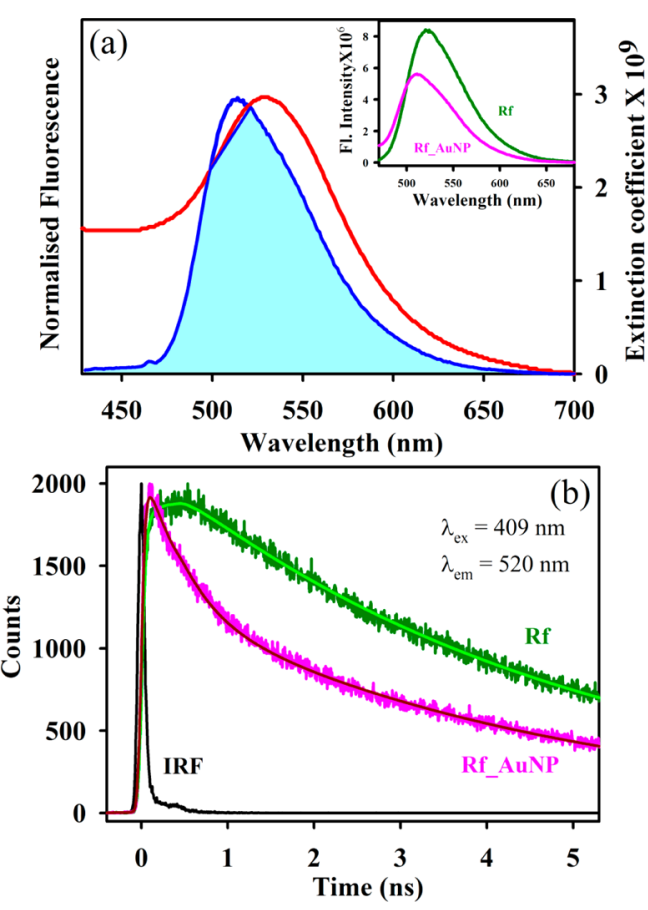

Figure 6. (a) Spectral overlap of donor Rf (dark green) and acceptor AuNPs (pink). Inset shows the steady-state emission quenching of Rf after complexation with AuNP. (b) The picosecond-resolved fluorescence transients of $\mathrm{Rf}$ and $\mathrm{Rf}-\mathrm{AuNPs}$ (excitation at $409 \mathrm{~nm}$ ). All the spectra were taken in aqueous medium.

illustrates a large spectral overlap of Rf emission and surface plasmon absorbance of Au NP which indicates a fair possibility of energy transfer from Rf to Au NP. The fluorescence intensity of the Rf is quenched and also the peak is shifted by $12 \mathrm{~nm}$ when it is attached to Au NPs in aqueous medium as shown in Figure 6a, inset. However, due to the change in the polarity of the solvent, riboflavin emission is shifted from $515 \mathrm{~nm}$ in ethanol (Figure 2b) to $522 \mathrm{~nm}$ in water. The fluorescence decay time of the $\mathrm{Rf}$ in water without AuNP is given by a single exponential with the average lifetime value of $4.76 \mathrm{~ns}$. However, there is a quenching of decay time in Rf-AuNP and the time scales are fitted by biexponential decay. The details of the fitting parameters of the fluorescence lifetime are given in Table 1 . The emission transient of $\mathrm{Rf}$ in water is fitted with single exponential decay of time constant $4.76 \mathrm{~ns}$. The emission of $\mathrm{Rf}-\mathrm{AuNPs}$ in water is quenched compared to that of the Rf. The associated fluorescence transient of $\mathrm{Rf}-\mathrm{AuNPs}$ is fitted biexponentially with one faster component of $0.4 \mathrm{~ns}$ (48\%) due to the energy transfer from $\mathrm{Rf}$ to AuNPs and a longer component of $4.65 \mathrm{~ns}$ (52\%) consistent with the decay of $\mathrm{Rf}$ only. The scheme of Förster's Resonance Energy-Transfer (FRET) is employed for the determination of the donoracceptor distance using eq 2 . The energy transfer efficiency and the overlap integral $[J(\lambda)]$ are calculated to be $53.7 \%$ from eq 3 and $1.36 \times 10^{20} \mathrm{M}^{-1} \mathrm{~cm}^{-1} \mathrm{~nm}^{4}$ from eq 1 , respectively. The Förster radius $\left(r_{0}\right)$ is found to be $34 \mathrm{~nm}$. From eq 2 , the calculated donor-acceptor separation $(r)$ is $28 \mathrm{~nm}$. As the estimated distance between the donor-acceptor exceeds $100 \AA$, the phenomenon of $\mathrm{Au}$ NPs based surface energy transfer (SET) process is a convenient spectroscopic ruler for longdistance measurement, which follows $1 / d^{4}$ distance dependence. NP-induced lifetime modification serves as a ruler to unravel the distance range well beyond $10 \mathrm{~nm} .{ }^{25}$ This kind of long distance SET from fluorescent dyes to Au NPs is well documented in the literature. In order to approve the NSET formulism, the distance between donor Rf and acceptor Au NP is determined to be $16.71 \mathrm{~nm}\left(d_{0}=17.36 \mathrm{~nm}\right)$ from eqs 6 and 7 , respectively. Herein, we propose nanosurface energy transfer (NSET) from the donor Rf to the acceptor AuNPs as the calculated donor-acceptor distance is in consonance with the size of the gold NPs (radius $15 \mathrm{~nm}$ ). Hence, it is worth emphasizing that the energy transfers from $\mathrm{Rf}$ to Au NP results in the reduced antioxidant activity of the Rf-AuNP nanohybrids as shown in Figure $4 \mathrm{~b}$.

\section{CONCLUSION}

We have conducted an in-depth investigation of the crucial photoinduced dynamics in riboflavin upon complexation with various inorganic nanoparticles (NPs) such as zinc oxide $(\mathrm{ZnO})$, titanium oxide $\left(\mathrm{TiO}_{2}\right)$, aluminum oxide $\left(\mathrm{Al}_{2} \mathrm{O}_{3}\right)$, and gold NPs of approximately $30 \mathrm{~nm}$ sizes. Raman spectroscopic studies reveal that riboflavin is adsorbed on the surface of the semiconductor $\left(\mathrm{TiO}_{2}\right.$ and $\left.\mathrm{ZnO}\right)$ and the insulator $\left(\mathrm{Al}_{2} \mathrm{O}_{3}\right) \mathrm{NPs}$ whereas a covalent attachment is observed with metal (gold). 2, 2-diphenyl-1-picrylhydrazyl (DPPH) assay under visible light irradiation was conducted to prove that semiconductors with a wide band gap show a more enhanced photoinduced antioxidant activity than insulators. Furthermore, the antioxidant activity is decreased in the gold nanohybrids as the electron is not shuttled to the model oxidant (DPPH). Timeresolved fluorescence studies on $\mathrm{Rf}-\mathrm{ZnO}$ and $\mathrm{Rf}-\mathrm{TiO}_{2}$ nanohybrids unravel the efficient electron transfer from photoexcited $\mathrm{Rf}$ to $\mathrm{ZnO}$ and $\mathrm{TiO}_{2}$. To confirm the energy transfer of the riboflavin to AuNPs, we have employed picosecond-resolved fluorescence studies and found that nano surface energy transfer (NSET) is predominant over Förster's 
resonance energy-transfer (FRET). NSET from Rf to Au NPs occur due to strong spectral overlap of $\mathrm{Rf}$ emission and surface plasmon absorption of the gold NP which is responsible for low antioxidant activity. The result of the study of photoinduced dynamics in the $\mathrm{Rf}$-nanohybrids could be incorporated in designing more effective photodynamic therapeutic agents.

\section{AUTHOR INFORMATION}

\section{Corresponding Author}

*E-mail: skpal@bose.res.in (S.K.P.). Telephone: +91 0332335 5706-08. Fax: +91 03323353477.

\section{Notes}

The authors declare no competing financial interest.

\section{ACKNOWLEDGMENTS}

S.C. thanks the CSIR (India) for the research fellowship. We thank DST (India) for financial grants DST/TM/SERI/2k11/ 103 and SB/S1/PC-011/2013. We also thank DAE (India) for financial grant 2013/37P/73/BRNS. We acknowledge Dr. A. Singha, Bose Institute for helping in Raman spectroscopic studies. P.L. acknowledges support by the NTH-School "Contacts in Nanosystems: Interactions, Control and Quantum Dynamics”, the Braunschweig IGSM, and DFG-RTG 1953/1.

\section{REFERENCES}

(1) Barton-Wright, E. C.; Moran, T.; Sarson, H. S. Riboflavin and Vitamin $B_{1}$ In Nineteenth Century Buns and Ale. Nature 1943, 152, 273.

(2) Huang, R.; Kim, H. J.; Min, D. B. Photosensitizing Effect of Riboflavin, Lumiflavin, and Lumichrome on the Generation of Volatiles in Soy Milk. J. Agric. Food Chem. 2006, 54, 2359-2364.

(3) Chaudhuri, S.; Batabyal, S.; Polley, N.; Pal, S. K. Vitamin $B_{2}$ in Nanoscopic Environments under Visible Light: Photosensitized Antioxidant or Phototoxic Drug? J. Phys. Chem. A 2014, 118, 39343943.

(4) Tsugita, A.; Okada, Y.; Uehara, K. Photosensitized Inactivation of Ribonucleic Acids in the Presence of Riboflavin. Biochim. Biophys. Acta 1965, 103, 360-363.

(5) Bernal, G. M.; LaRiviere, M. J.; Mansour, N.; Pytel, P.; Cahill, K. E.; Voce, D. J.; Kang, S.; Spretz, R.; Welp, U.; Noriega, S. E.; et al. Convection-Enhanced Delivery and In Vivo Imaging of Polymeric Nanoparticles for the Treatment of Malignant Glioma. Nanomed. Nanotechnol. Biol. Med. 2014, 10, 149-157.

(6) Huang, R.; Choe, E.; Min, D. B. Kinetics for Singlet Oxygen Formation by Riboflavin Photosensitization and the Reaction between Riboflavin and Singlet Oxygen. J. Food Sci. C: Food Chem. Toxicol. 2004, 69, C726-C732.

(7) de La Rochette, A.; Silva, E.; Birlouez-Aragon, I.; Mancini, M.; Edwards, A. M.; Morliere, P. Riboflavin Photodegradation and Photosensitizing Effects are Highly Dependent on Oxygen and Ascorbate Concentrations. Photochem. Photobiol. 2000, 72, 815-820.

(8) Wollensak, G.; Spoerl, E.; Seiler, T. Riboflavin/Ultraviolet-AInduced Collagen Crosslinking for the Treatment of Keratoconus. Am. J. Ophthalmol. 2003, 135, 620-627.

(9) Kashiwabuchi, R. T.; Khan, Y.; Carvalho, F. R. d. S.; Hirai, F.; Campos, M. S.; McDonnell, P. J. Antimicrobial Susceptibility of Photodynamic Therapy (UVA/Riboflavin) against Staphylococcus aureus. Arq. Bras. Oftalmol. 2012, 75, 423-426.

(10) Petros, R. A.; DeSimone, J. M. Strategies in the Design of Nanoparticles for Therapeutic Applications. Nat. Rev. Drug Discovery 2010, 9, 615-627.

(11) Zhang, L.; Gu, F. X.; Chan, J. M.; Wang, A. Z.; Langer, R. S.; Farokhzad, O. C. Nanoparticles in Medicine: Therapeutic Applications and Developments. Clin. Pharmacol. Ther. 2008, 83, 761-769.
(12) Bechet, D.; Couleaud, P.; Frochot, C.; Viriot, M. L.; Guillemin, F.; Barberi-Heyob, M. Nanoparticles as Vehicles for Delivery of Photodynamic Therapy Agents. Trends Biotechnol. 2008, 26, 612-621.

(13) Cheng, C. J.; Tietjen, G. T.; Saucier-Sawyer, J. K.; Saltzman, W. M. A Holistic Approach to Targeting Disease with Polymeric Nanoparticles. Nat. Rev. Drug Discovery 2015, DOI: 10.1038/nrd4503.

(14) Davis, M. E.; Chen, Z.; Shin, D. M. Nanoparticle Therapeutics: An Emerging Treatment Modality for Cancer. Nat. Rev. Drug Discovery 2008, 7, 771-782.

(15) Mishra, B.; Patel, B. B.; Tiwari, S. Colloidal nanocarriers: A review on Formulation Technology, Types and Applications Toward Targeted Drug Delivery. Nanomedicine. 2010, 6, 9-24.

(16) Xiong, H.-M. ZnO Nanoparticles Applied to Bioimaging and Drug Delivery. Adv. Mater. 2013, 25, 5329-5335.

(17) Sardar, S.; Chaudhuri, S.; Kar, P.; Sarkar, S.; Lemmens, P.; Pal, S. K. Direct Observation of Key Photoinduced Dynamics in a Potential Nano-Delivery Vehicle of Cancer Drugs. Phys. Chem. Chem. Phys. 2015, 17, 166-177.

(18) Shang, Y.; Min, C.; Hu, J.; Wang, T.; Liu, H.; Hu, Y. Synthesis of Gold Nanoparticles by Reduction of HAuCl4 under UV Irradiation. Solid State Sci. 2013, 15, 17-23.

(19) Storhoff, J. J.; Elghanian, R.; Mucic, R. C.; Mirkin, C. A.; Letsinger, R. L. One-Pot Colorimetric Differentiation of Polynucleotides with Single Base Imperfections Using Gold Nanoparticle Probes. J. Am. Chem. Soc. 1998, 120, 1959-1964.

(20) Duyvis, M. G.; Hilhorst, R.; Laane, C.; Evans, D. J.; Schmedding, D. J. Role of Riboflavin in Beer Flavor Instability: Determination of Levels of Riboflavin and Its Origin in Beer by Fluorometric Apoprotein Titration. J. Agric. Food. Chem. 2002, 50, $1548-1552$.

(21) Liu, X.; Atwater, M.; Wang, J.; Huo, Q. Extinction Coefficient of Gold Nanoparticles with Different Sizes and Different Capping Ligands. Colloids Surf., B 2007, 58, 3-7.

(22) Sinha, S. S.; Verma, P. K.; Makhal, A.; Pal, S. K. A Versatile Fiber-Optic Coupled System for Sensitive Optical Spectroscopy in Strong Ambient Light. Rev. Sci. Instrum. 2009, 80, 053109-053104.

(23) Lakowicz, J. R. Principles of Fluorescence Spectroscopy. Kluwer Academic/Plenum: New York. 2006.

(24) Sun, M.; Moore, T. A.; Song, P. S. Molecular Luminescence Studies of Flavins. I. The Excited States of Flavins. J. Am. Chem. Soc. . 1972, 94, 1730-1740.

(25) Muhammed, M. A. H.; Shaw, A. K.; Pal, S. K.; Pradeep, T. Quantum Clusters of Gold Exhibiting FRET. J. Phys. Chem. C 2008, 112, 14324-14330.

(26) Persson, B. N. J. Electron-Hole-Pair Quenching of Excited States Near a Metal. Phys. Rev. B 1982, 26, 5409-5415.

(27) Craig, D. P.; Thirunamachandran, T. Molecular Quantum Electrodynamics: An Introduction to Radiation-Molecule Interactions; Courier Dover Publications: Mineola, NY, 1998.

(28) Sardar, S.; Sarkar, S.; Myint, M. T. Z.; Al-Harthi, S.; Dutta, J.; $\mathrm{Pal}, \mathrm{S} . \mathrm{K}$. Role of Central Metal Ions in HematoporphyrinFunctionalized Titania in Solar Energy Conversion Dynamics. Phys. Chem. Chem. Phys. 2013, 15, 18562-18570.

(29) Reller, A.; Cocke, D. L. High Resolution Transmission Electron Microscopic (HRTEM) Determination of the Preferentially Exposed Faces on $\gamma-\mathrm{Al}_{2} \mathrm{O}_{3}$ and $\eta-\mathrm{Al}_{2} \mathrm{O}_{3}$. Catal. Lett. 1989, 2, 91-95.

(30) Vaishnavi, E.; Renganathan, R. Photochemical Events during Photosensitization of Colloidal $\mathrm{ZnO}$ Nanoparticles by Riboflavin. Bull. Mater. Sci. 2012, 35, 1173-1179.

(31) Daniel, M.-C.; Astruc, D. Gold Nanoparticles: Assembly, Supramolecular Chemistry, Quantum-Size-Related Properties, and Applications toward Biology, Catalysis, and Nanotechnology. Chem. Rev. 2004, 104, 293-346.

(32) De Gelder, J.; De Gussem, K.; Vandenabeele, P.; Moens, L. Reference Database of Raman Spectra of Biological Molecules. J. Raman Spectrosc. 2007, 38, 1133-1147.

(33) Chat, O. A.; Najar, M. H.; Dar, A. A. Evaluation of Reduction Kinetics of 2,2-Diphenyl-1-Picrylhydrazyl Radical by Flavonoid 
Glycoside Rutin in Mixed Solvent Based Micellar Media. Colloids Surf., A 2013, 436, 343-353.

(34) Arce, V. B.; Scotto, J.; Allegretti, P. E.; Melo, M. A.; Airoldi, C.; Salum, M. L.; Erra-Balsells, R.; Pis Diez, R.; Mártire, D. O. Combined Experimental and Computational Investigation of the Fluorescence Quenching of Riboflavin by Cinnamic Alcohol Chemisorbed on Silica Nanoparticles. J. Phys. Chem. C 2014, 118, 15348-15355.

(35) Escalada, J. P.; Arce, V. B.; Porcal, G. V.; Biasutti, M. A.; Criado, S.; García, N. A.; Mártire, D. O. The Effect of Dichlorophen Binding to Silica Nanoparticles on Its Photosensitized Degradation in Water. Water Res. 2014, 50, 229-236.

(36) Escalada, J. P.; Arce, V. B.; Carlos, L.; Porcal, G. V.; Biasutti, M. A.; Criado, S.; Garcia, N. A.; Martire, D. O. Photodegradation Routes of the Herbicide Bromoxynil in Solution and Sorbed on Silica Nanoparticles. Env. Sci. Process. Impact. 2014, 16, 858-865. 